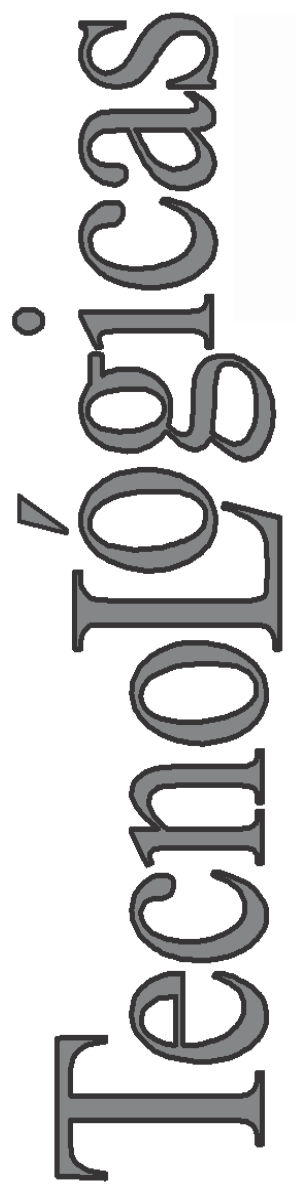

\title{
Confrontación Técnica de dos Prototipos de Secado Dieléctrico de Madera usando Radiofrecuencia
}

\section{Technical Confrontation of Two Prototypes of Dielectric Drying Wood using Radio Frequency}

\author{
Willer F. Montes-Granada ${ }^{1}$ \\ Sara M. Yepes-Zuluaga ${ }^{2}$
}

1 Departamento de Electrónica y Telecomunicaciones, Instituto Tecnológico Metropolitano, Medellín-Colombia willermontes@itm.edu.co

2 Departamento de Electrónica y Telecomunicaciones, Instituto Tecnológico Metropolitano, Medellín-Colombia sarayepes@itm.edu.co 


\section{Resumen}

Partiendo de la premisa de que el secado endógeno dieléctrico es el que mejores resultados proporciona al aplicarlo a madera, se confrontaron dos prototipos diferentes desde las perspectivas de la filosofía de diseño de la tecnología de $50 \Omega$ y la de bomba de calor; de los resultados obtenidos en un proceso de secado para una probeta de madera, se identificó que el prototipo implementado con tecnología de $50 \Omega$ obtuvo mejores resultados con respecto a la eficiencia del proceso de secado. Este trabajo abre la brecha a futuras investigaciones en torno a este tipo de aplicaciones industriales de la Radiofrecuencia (RF), con miras al diseño a escala de un prototipo de horno industrial de bajo costo para el secado de madera.

\section{Palabras clave}

Radiofrecuencia (RF); secado endógeno de madera; secado dieléctrico; secado no convencional; prototipo experimental.

\section{Abstract}

Starting from the premise that dielectric endogenous drying method is the one that gives better results on wood, there will be a confrontation between two different prototypes from the perspectives of the design philosophy and outcome results in a wooden probe. This work opens the gap for future research around this type of industrial RF applications, in order to design a prototype scale industrial furnace for wood drying.

\section{Keywords}

Radiofrequency (RF); endogenous drying wood; dielectric drying; unconventional drying; experimental prototype. 


\section{INTRODUCCIÓN}

Por ser la madera un material orgánico, después de talada su degradación puede ser muy rápida; para preservarla el método más importante es el secado, ya que controla sus contenidos de humedad optimizando su posterior procesamiento. Además, las empresas de transporte maderero terrestre deben cumplir con los requisitos especificados en la Norma Internacional Fitosanitaria NIMF No.15 (CIPF, 2002), que establece que antes de movilizar la madera debe pre-secarse por un lapso de media hora a una temperatura de $56^{\circ} \mathrm{C}$, lo cual no solo previene su deterioro natural sino que evita la supervivencia de algunas especies de hongos, insectos xilófagos y otros parásitos inherentes a la humedad (Pedras, 2004). Fuera de la preservación, el secado se requiere para obtener las características necesarias para ser la materia prima de un sinnúmero de productos, ya que incrementa sus propiedades estructurales y de resistencia física, así como la estabilidad de sus propiedades eléctricas y de aislamiento térmico; sin embargo, de no manejarse adecuadamente conlleva procesos de transferencia de masa y calor que pueden producir distribuciones no uniformes de humedad y temperatura, repercutiendo en la generación de defectos de secado (Ananías, 2008; FPL, 2010). Consecuentemente, el método de secado y el proceso utilizado en cada uno de ellos influirán directamente en la calidad y duración del producto final.

\subsection{Métodos de Secado para Madera}

Los tres métodos usados son: secado natural, secado en hornos o cámaras y secado híbrido. El natural consiste en acopiar la madera en bodegas con circulación de aire natural o forzado, para secarla por extracción y difusión de humedad libre. El secado en hornos consiste en introducir la madera en equipos diseñados para obtener calentamiento de la madera e indirectamente reducir su contenido de humedad $(\mathrm{CH})$; pueden ser exógenos o aparatos mecánicos alimentados a partir de combustibles gaseosos, fósiles y subproductos del proceso, que aunque mejoran los tiempos del secado natural provocan contaminación por emisión de partículas y $\mathrm{CO}_{2}$, así como un gran deterioro de la calidad final del material, 
produciéndose defectos como agrietamientos, manchas y cambios de coloración, colapsos y estrés, alabeos y torceduras, sin lograr un gran impacto en la eficiencia del proceso (Denig et al, 2000; FPL, 2010); también los hay endógenos, hornos o cámaras de secado que operan a partir de electricidad, en los que ocurre calentamiento dieléctrico por medio de energía de alta frecuencia (HF), que además de no contaminar el medio ambiente permiten obtener un mínimo deterioro del material y tiempos de secado, ya que la longitud de onda de los campos Electromagnéticos (EM) generados permiten variar la profundidad de penetración dentro de la madera proporcionando un secado homogéneo, de adentro hacia afuera, similar a como lo hacen los hornos para alimentos (Denig et al, 2000). El secado híbrido es una mezcla de los dos anteriores; se centra en obtener un pre-secado con aire (natural o forzado) hasta cerca del punto de saturación de la fibra (psf) y completar el proceso en un horno; algunos de estos equipos son sofisticados y requieren de etapas especiales para extraer los residuos del proceso (como vapor de agua) u obtener vacío.

Para la elección del método idóneo las dos condiciones relevantes son: obtener el porcentaje de humedad adecuado en el material (en función de su potencial aplicación) y obtener una distribución volumétricamente uniforme de ella; para esto deben tenerse en cuenta factores como la especie y el tamaño de la pieza de madera, la humedad inicial y la requerida, la tasa relativa de secado y la degradación o daño permisible (FPL, 2010).

En Colombia, la poca tecnificación en el sector forestal genera un impacto ambiental negativo y un incremento en los costos de producción; el secado natural es el más popular, quizás por su aparente economía, pero en realidad es un método ineficiente porque produce pérdidas de materia prima hasta de un $15 \%$ y consume gran tiempo (entre 25 y 40 días) para obtener un $\mathrm{CH}$ cercano a $20 \%$, muy alto para la mayoría de aplicaciones comerciales de la madera (Denig et al., 2000; FPL, 2010). Por esto, se hace necesario el trabajo investigativo en métodos eficientes de secado endógeno, en los que se provea al proceso de características especiales, como el incremento de la difusión de vapor y humedad, correlación entre los gradientes de temperatura y concentración de masa y estabilización de la temperatura por debajo del punto de 
ebullición (Denig et al., 2000). Según Kudra y Mujumdar (2009), su principal ventaja es extraer la humedad volumétricamente uniforme, por lo que resulta óptima la calidad del producto final; su desventaja radica en los elevados costos de implementación tecnológica y costos energéticos de operación.

\subsection{Tecnologías de Secado Endógeno por RF}

Tecnología de $50 \Omega$ : es una técnica avanzada que provee continua sintonización del generador con la carga por ajuste de amplitud o fase de la señal, manteniendo fija la frecuencia de operación y estabilizando la impedancia de salida en $50 \Omega$, lo que permite optimizar la energía EM y obtener una mayor uniformidad en el secado; opera típicamente en frecuencias de RF de 13,56 y 27,12 MHz (Kudra \& Mujumdar, 2009).

Radio Frecuencia asistida por bomba de calor: incrementa la velocidad de transferencia de calor usando una bomba dieléctrica, lo que acelera el proceso de secado y permite evaporar el agua sin exceder los $82^{\circ} \mathrm{C}$. (Marshall \& Metaxas, 1999). Como desventaja se tiene que pueden darse diferentes resultados dependiendo de la especie u otras características especiales de la madera a secar, ya que los materiales no polares como la grasa, el aceite y los sólidos secos que forman gran parte del cuerpo de la madera no reaccionan efectivamente a dichos campos EM (Kudra \& Mujumdar, 2009).

Secado híbrido RF y Vacío (RF/V): permite incrementar la eficiencia energética obteniendo las ventajas del calentamiento dieléctrico, la fácil evacuación del vapor al vacío y la posibilidad de apilar la madera a secar en rastreles, lo que reduce el volumen de carga y evita arqueos, torceduras y otros defectos en ella. La baja temperatura de secado (entre $30^{\circ} \mathrm{C}$ y $40^{\circ} \mathrm{C}$ ) obtenida como resultado de un reducido punto de ebullición, hace que la madera conserve su resistencia mecánica y sea menos susceptible a los defectos producidos por el proceso, permitiendo teóricamente un decremento de humedad desde un $90 \%$ hasta un $8 \%$, con una eficiencia de secado de hasta 20 veces mayor que en un horno convencional 
(Kudra \& Mujumdar, 2009). Su gran desventaja radica en que utilizan frecuencias de microondas, lo cual no sólo hace necesario el uso de dispositivos tipo klynstron, magnetrones y cavidades resonantes, sino también de cámaras de secado a manera de guías de onda; esto hace que sean voluminosos y costosos además de consumir gran cantidad de energía eléctrica, características que los hacen viables sólo para grandes productores de madera (Sanga et al, 2000).

El secado dieléctrico ha demostrado ser la técnica más eficiente para la madera, en cuanto a los bajos contenidos de humedad final que logra y a la excelente calidad del producto obtenido. En este trabajo se confrontan dos prototipos para experimentación en este tipo de secado, con filosofías de diseño diferentes correspondientes a las dos primeras tecnologías, desde la perspectiva de sus bloques funcionales constitutivos y de los resultados obtenidos para un proceso de secado de una pieza de madera de iguales características; se enfoca el trabajo en esta dirección pues se prevé que son las técnicas de más bajo costo de implementación tecnológica, y que por tanto aplicarían en nuestro contexto a la hora de producir un horno industrial teniendo como público objetivo el pequeño y mediano productor de madera.

\subsection{Prototipo Implementado}

Se diseñó e implementó un prototipo para experimentación en secado dieléctrico basado en una tecnología de $50 \Omega$ con algunas variantes, entre las que sobresale la frecuencia de operación de $40,7 \mathrm{MHz}$, superior a las que típicamente se trabajan (Montes, 2012). Los acabados finales del prototipo con sus paneles frontal y trasero se ilustran en la Fig. 1.

El diagrama de bloques funcional, ilustrado en la Fig. 2, está constituido esencialmente de un generador de RF, un dispositivo acoplador de impedancias entre generador y carga, una plataforma de secado y otros aditamentos auxiliares para protecciones, control eléctrico y maniobra del material. 

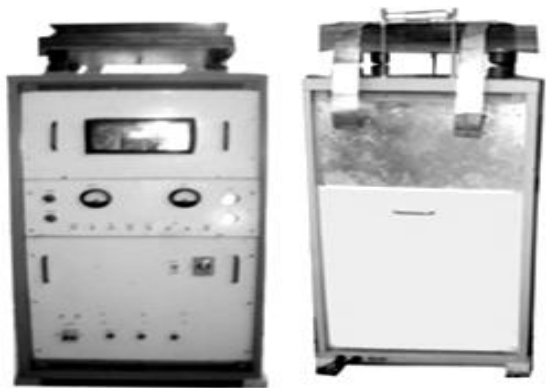

Fig. 1. Acabado final del prototipo implementado. Fuente: (Montes, 2012)

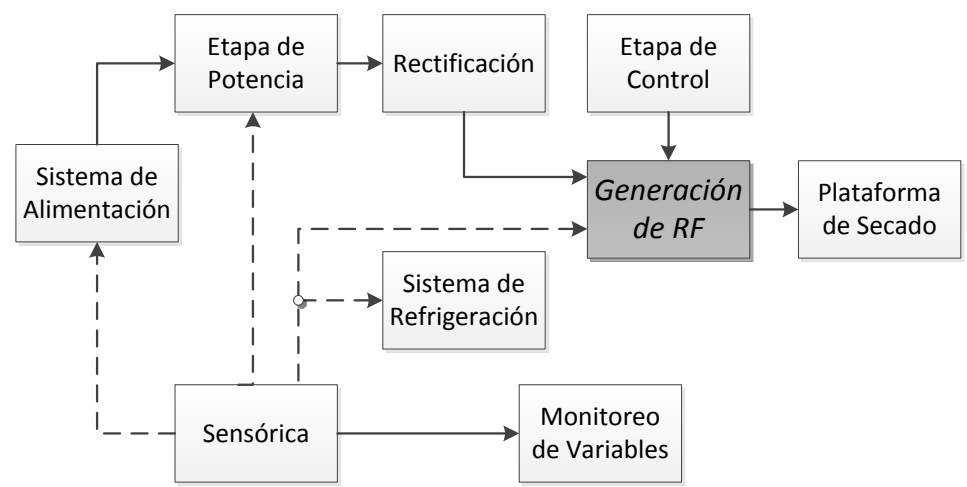

Fig. 2. Diagrama de bloques del prototipo implementado. Fuente: Autores

\section{METOdOLOGía}

Luego del proceso de diseño e implementación del sistema propuesto, donde se validó el fenómeno de calentamiento endógeno dieléctrico y se logró el secado de madera con resultados satisfactorios (Montes, 2012), se quiso confrontar con otra tecnología de igual naturaleza pero con diferente filosofía de diseño. Se tomó un trabajo de investigación basado en una tecnología de RF asistida por bomba de calor, que tenía la posibilidad de realizar tres variantes en el método de secado: por conducción de agua caliente, por descargas de alta frecuencia, y por la combinación de los dos anteriores (Hongda \& Fengxian, 2012); posteriormente se obtuvo un diagrama de bloques funcional presentado en la Fig. 3, a partir 
de la descripción del dispositivo experimental que allí se trabajó con la finalidad de poder cualificar de manera general el costo de implementación de cada prototipo. Además, se realizó un proceso experimental de secado similar al propuesto en la metodología empleada por Hongda y Fengxian (2012), para evaluar la eficiencia del proceso en cuanto a tiempo requerido para obtener un porcentaje de $\mathrm{CH}$ mínimo, para una probeta de madera de iguales especificaciones.

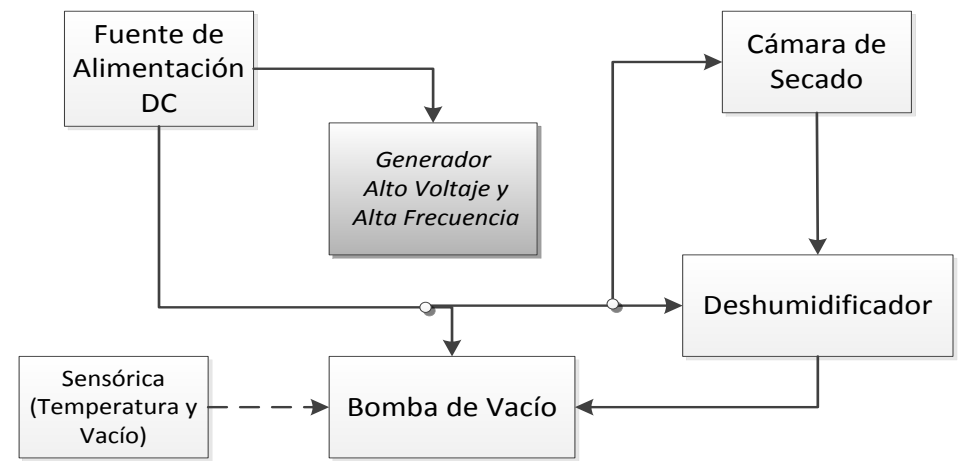

Fig. 3. Diagrama de bloques del prototipo confrontado. Fuente: Autores

\section{RESULTADOS Y DISCUSIÓN}

\subsection{Confrontación de Bloques Constitutivos}

Ambos prototipos tienen las etapas básicas de Fuentes de alimentación que proveen la energía necesaria para las etapas activas, tanto $\mathrm{DC}$ como $\mathrm{AC}$; también lo correspondiente al monitoreo de variables, teniendo en cuenta que la tecnología de $50 \Omega$ (Fig. 2) monitorea las variables eléctricas de operación del dispositivo de potencia de RF, mientras que el de bomba de calor (Fig.3) las variables de temperatura y vacío dentro de la cámara durante el proceso de secado. La etapa de generación de RF para la Fig. 2 opera con una frecuencia ISM de $40,7 \mathrm{MHz}$ sinusoidal, y permite trabajar con potencias inferiores a $400 \mathrm{~W}$ y $3000 \mathrm{~V}$; en la etapa de generación de la Fig.3 se opera con pulsos de frecuencia de $50 \mathrm{KHz}$, potencia de $120 \mathrm{~W}$ y $2000 \mathrm{~V}$. Las principales diferencias se concen- 
tran en la cámara de secado de cada prototipo, donde el de la Fig. 2 se limita a una plataforma de secado capacitiva externa y en la Fig. 3 se requiere aditamentos especializados como etapas de deshumidifación y bomba de vacío.

\subsection{Confrontación Proceso de Secado}

Las pruebas realizadas consistieron en someter a secado una pieza de abedul de 50*150*350 $\mathrm{mm}$ en el prototipo implementado de la Fig.2, con un contenido de humedad $(\mathrm{CH})$ inicial de $95 \%$, medido por un método gravimétrico recomendado donde se requiera una buena calidad de secado final, en especies de difícil secado sensibles a las condiciones ambientales y que requieren precisión para establecer programas de secado y cuando se desconoce el comportamiento de una especie o se carece de la información necesaria para su conducción (FPL, 2010). Este consiste en determinar la evolución de la humedad de la madera para diferentes tiempos, mediante la extracción y pesada de probetas de unos $5 \mathrm{~cm}$ (testigos) de piezas representativas de la tabla (centro y esquinas); para cada tiempo se tomaron tres probetas y se pesaron con una aproximación de $\pm 0.1 \mathrm{gr}$, se promediaron los valores y se registraron. Para obtener el peso anhidro (peso seco), se promedió el peso de tres probetas secadas en un horno exógeno a $102 \pm 2^{\circ} \mathrm{C}$. Tras obtener el peso húmedo y seco de las probetas se calculó $\mathrm{CH}$ para cada tiempo, por medio de la ecuación (1).

$C H(\%)=\frac{P_{H}-P_{S}}{P_{S}} * 100 \%$

Donde, $\mathrm{P}_{\mathrm{H}}$ : Peso húmedo o Peso Inicial de la madera y PS: Peso seco o anhidro. Al gráfico del proceso experimental aportado por Hongda y Fengxian (2012), se le insertan los datos de secado con el prototipo de tecnología de $50 \Omega$, para obtener la gráfica comparativa ilustrada en la Fig. 4 y proceder a la confrontación de resultados. 


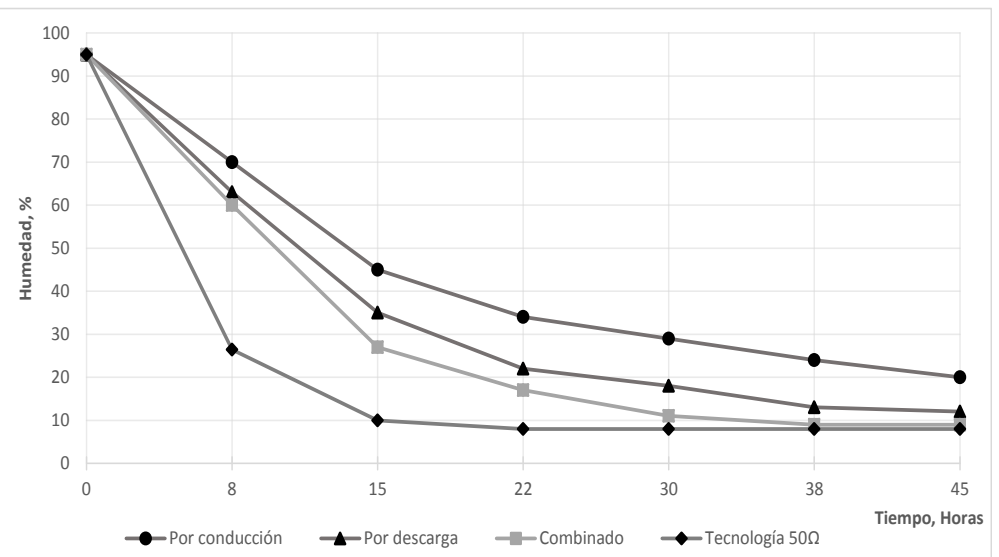

Fig. 4. Curvas de secado de los procesos experimentales. Fuente: Autores

Se diferencia claramente las dos etapas características del proceso de secado de que hablan los autores: la fase de velocidad de secado constante y la fase de velocidad de secado decreciente (Fernández-Golfin et al., 2007; Ananías, 2008). La primera fase, se presenta al inicio del secado cuando la madera se encuentra completamente húmeda y su superficie está cubierta de una delgada película de agua líquida, por lo que la evaporación se da a una velocidad constante; en el proceso de secado con el prototipo de 50 $\Omega$ se dio aproximadamente para un rango de tiempo entre 0 y 8 horas, con humedades entre $95 \%$ y $25 \%$. En contraste, para los resultados experimentales del prototipo a bomba de calor se obtuvieron entre 0 y 15 horas, con diferentes rangos de humedades, así: entre 95 y $46 \%$ para el método de conducción; entre 95 y $35 \%$ para el método de descarga; entre 95 y $27 \%$ para el método que combina los dos anteriores.

En la segunda fase se observan dos sub-etapas: la de secado con superficie parcialmente cubierta de agua líquida, período que se produce cuando aparecen los primeros sectores volumétricamente secos y que puede prolongarse hasta cerca del punto de saturación de las fibras (psf); para este proceso se dio entre 8 y 15 horas permitiendo humedades entre $25 \%$ y $9 \%$, para la tecnología de $50 \Omega$. Para la otra tecnología se dio entre 15 y 22 horas, permi- 
tiendo rangos de humedades, así: entre 46 y $34 \%$ para el método de conducción; entre 35 y $22 \%$ para el método de descarga; entre 27 y 18\% para el método combinado. La sub-etapa por debajo del psf, gobernada por el movimiento interno de humedad representa la mayor parte de la curva de secado y se dio estable en $8 \%$ a par-

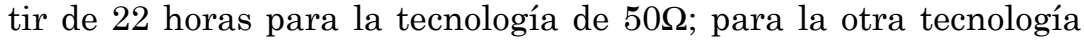
se veía una tendencia de decremento, sin estabilizarse aún para las 45 horas de prueba.

\section{CONCLUSIONES}

De la confrontación de los bloques constitutivos de ambas tecnologías se puede inferir que el prototipo de $50 \Omega$ tendría un menor costo de implementación debido a la plataforma de secado seleccionada, ya que no requiere mecanismos para la evacuación de productos del proceso como agua, vapor y posibles gases emanados. De la confrontación del proceso de secado se concluye que el prototipo de $50 \Omega$ propuesto lleva a una $\mathrm{CH}$ final de $10 \%$ en un tiempo de tan solo 15 horas; para el dispositivo de bomba de calor se logran estos resultados para un tiempo de 45 horas para la variante combinada, de 60 horas para el de descarga y 90 horas para el de conducción. Esto comprueba la gran eficiencia del prototipo propuesto.

Como trabajo futuro se proyectan pruebas de consumo eléctrico para determinar el costo real de un proceso de secado; esto permitirá evaluar la viabilidad de competir con tecnologías de secado exógeno de mayor demanda en el sector forestal, no solo con la calidad del producto final sino también en costos operativos.

\section{AGRADECIMIENTOS}

Este trabajo fue soportado por el grupo de investigación Automática y Electrónica (A\&E) del Instituto Tecnológico Metropolitano-ITM, dentro del proyecto P13119. 


\section{REFERENCIAS}

Ananías, R. (2008). Física de la madera. Departamento de Ingeniería en Maderas. Univer-sidad del BioBio - UBB, Chile.

Denig, J.; Wengert, E.M.; Simpson, W.T. (2000). Drying Hardwood Lumber. United States Department of Agriculture - USDA. Forest Products Laboratory, General-Technical Report FPL-GTR-118. Madison, Wisconsin.138 p.

Fernández-Golfín S., J.I. y Conde G., M. (2007). Manual Técnico de Secado de Maderas. Escuela Técnica Superior de Ingenieros de Montes, Universidad Politécnica de Ma-drid. Edita AITIM. ISBN 10:84-8738137-5. $252 \mathrm{pp}$.

FPL - Forest Products Laboratory. (2010). Wood Handbook: Wood as an Engineering Material. General Technical Report FPL-TR-190. U.S. Forest Service, Forest Prod-ucts Laboratory. Centennial Edition. Madison, Wisconsin. 508 p.

Kudra, T., and Mujumdar, A.S. (2009). Advanced Drying Technologies. CRC Press, 2nd ed. ISBN 978-1-4200-7387. New York, pp. 397-417.

Hongda, L., and Fengxian L. (2012). "Wood Drying by High Frequency Discharge", Power and Energy Engineering Conference (APPEEC), pp.1-3.

Montes G., W.F. (2012). "Experimental prototype for endogenous drying wood by radio frequency (RF)", Alternative Energies and Energy Quality (SIFAE), pp.1-8.

Norma Internacional Fitosanitaria NIMF No.15. (2002). FAO, Convención Internacional de Protección Fitosanitaria - CIPF, Roma.

Pedras S., F. (2004). Programa de conformidad fitosanitaria de embalajes. Área de Innovación y Tecnología - Revista CIS: Centro de Innovación y Servicios Tecno-lógicos de la Madera, ISSN 1138-7726, № 12 , pp. 47-51. 